\title{
Long-term quality of life after laparoscopic distal gastrectomy for early gastric cancer: results of a prospective multi-institutional comparative trial
}

\author{
Kazunari Misawa $\cdot$ Michitaka Fujiwara $\cdot$ Masahiko Ando $\cdot$ Seiji Ito $\cdot$ Yoshinari Mochizuki $\cdot$ \\ Yuichi Ito $\cdot$ Eiji Onishi $\cdot$ Kiyoshi Ishigure $\cdot$ Yuki Morioka $\cdot$ Tsunenobu Takase $\cdot$ \\ Takuya Watanabe $\cdot$ Yoshitaka Yamamura $\cdot$ Satoshi Morita $\cdot$ Yasuhiro Kodera
}

Received: 7 September 2013/Accepted: 10 April 2014/Published online: 7 May 2014

(C) The International Gastric Cancer Association and The Japanese Gastric Cancer Association 2014

\begin{abstract}
Background The aim of this study was to compare the postoperative health-related quality of life (HRQOL) between open and laparoscopic distal gastrectomy.

Methods A multi-institutional nonrandomized study was conducted. Patients with clinical T1 gastric cancer were prospectively enrolled and underwent distal gastrectomy by either the open or laparoscopic approach. HRQOL was measured using the European Organization for Research and Treatment of Cancer, Quality of Life QuestionnaireCore 30 and the site-specific module for gastric cancer. Questionnaires were completed at baseline and at 1,3,6, and 12 months postoperatively. Clinicopathological characteristics and short-term outcome including postoperative morbidity and HRQOL were compared between the approaches.
\end{abstract}

K. Misawa $(\bowtie) \cdot S$. Ito $\cdot$ Y. Ito

Department of Gastroenterological Surgery, Aichi Cancer Center Hospital, 1-1 Kanokoden, Chikusa-ku, Nagoya 464-8681, Japan e-mail: misawakzn@aichi-cc.jp

\section{Fujiwara · Y. Kodera}

Department of Gastroenterological Surgery (Surgery II),

Nagoya University Graduate School of Medicine, Nagoya, Japan

M. Ando

Center for Advanced Medicine and Clinical Research,

Nagoya University Graduate School of Medicine, Nagoya, Japan

Y. Mochizuki

Department of Surgery, Komaki Municipal Hospital,

Komaki, Japan

E. Onishi · Y. Morioka

Department of Surgery, Ichinomiya Municipal Hospital,

Ichinomiya, Japan
Results A total of 145 patients (open, $n=72$; laparoscopic, $n=73$ ) were enrolled between September 2008 and January 2011 and analyzed. The laparoscopic approach was associated with longer operating time, less blood loss, and a similar incidence of postoperative complications. At each time point, the questionnaires were retrieved from more than $90 \%$ of the patients. The worst scores for most of the scales were observed at 1 month postoperatively and improved thereafter. No statistically significant differences were observed regarding physical functioning, the primary endpoint. On the other hand, role, emotional, cognitive, and social functioning scores were superior in the laparoscopic group at 6 and 12 months postoperatively. Symptom scales including fatigue, pain, eating restriction, taste problems, and anxiety were better in the laparoscopic group before 6 months but not at 12 months.

\author{
K. Ishigure \\ Department of Surgery, JA Konan Kosei Hospital, \\ Konan, Japan \\ T. Takase \\ Department of Surgery, JA Kainan Hospital, \\ Yatomi, Japan \\ T. Watanabe \\ Department of Surgery, Tokai Central Hospital, \\ Kakamigahara, Japan \\ Y. Yamamura \\ Department of Surgery, Nagoya Memorial Hospital, \\ Nagoya, Japan \\ S. Morita \\ Department of Biostatistics and Epidemiology, \\ Yokohama City University, Yokohama, Japan
}


Conclusions The study was considered to be negative because no benefit of the laparoscopic approach was observed in terms of physical functioning. However, more favorable scores for some of the symptom scales during the first 6 months and several functioning scales at 12 months after surgery suggest a potential benefit of the laparoscopic approach.

Keywords Quality of life - Questionnaire - Laparoscopic surgery · Gastrectomy · Gastric cancer

\section{Introduction}

The laparoscopic approach has been keenly pursued by surgeons who opt to provide patients with minimally invasive surgery, and the indication has been expanded to include oncological surgery. Several prospective randomized trials conducted for colon cancer have shown that the long-term outcome is not inferior when the laparoscopic approach was used [1,2]. Whether the same is true for gastric cancer surgery remains uncertain because of the greater propensity of gastric cancer cells to scatter into the peritoneal cavity and form deposits, the process being reportedly facilitated by abdominal inflation at the time of laparoscopic surgery. Given the paucity of resectable gastric cancers and expert laparoscopic surgeons devoted to gastric surgery in Western countries, randomized trials of sufficient sample size to make comparisons between the approaches have been conducted exclusively in the Far East. Despite such circumstances, the proportion of laparoscopic approach in gastric cancer surgery in general has increased exponentially during the past decade. In particular, clinically T1 stage gastric cancer tends to be an optimal target for this approach if it is considered to be ineligible for endoscopic submucosal dissection [3].

Regarding short-term outcome, the laparoscopic approach for distal gastric cancer is known for reduced blood loss, prolonged operating time, and lymph node retrieval and morbidity that are equivalent to the open approach [4-7]. Health-related quality of life (HRQOL) as evaluated by established instruments is another important outcome to be compared between the two approaches. In the current study, patient-reported outcome was evaluated and compared between the approaches using the Japanese language version of the European Organization for Research and Treatment of Cancer (EORTC), Quality of Life Questionnaire Core 30 (QLQ-C30) and QLQ-STO22, a module for evaluating gastric cancer patients [8-11]. The comparison was made among patients with early-stage gastric cancer who underwent distal gastrectomy with the Japanese guideline-oriented lymph node dissection [12]. The study was conducted by a group of hospitals that are affiliated to the Department of Gastroenterological Surgery, Nagoya University Graduate School of Medicine.

\section{Patients and methods}

This is a prospective nonrandomized phase II study of the difference in the patient-reported outcome between open and laparoscopic gastrectomy for early-stage gastric cancer. The primary endpoint was the longitudinal score of physical functioning as measured by the QLQ-C30 up to 12 months after surgery, and the secondary endpoints were the operating time, amount of blood loss, morbidity, and other subscales in QLQ-C30 and QLQ-ST22.

Patients who were eligible for the following criteria were registered: gastric adenocarcinoma confirmed by endoscopic biopsy; diagnosed as T1 through endoscopy, endoscopic ultrasonography, and computerized tomography; considered as resectable by either distal gastrectomy or pylorus-preserving gastrectomy (PPG); considered as sufficiently medically fit to undergo gastrectomy under general anesthesia; considered to have sufficient ability to fill in the EORTC questionnaires; and having given written consent after the nature of the study had been explained. Patients with secondary malignancy, those with body mass index (BMI) exceeding $28 \mathrm{~kg} / \mathrm{m}^{2}$, and those who needed co-resection of organs other than the gallbladder were deemed ineligible.

The patients were given a set of questionnaires and were asked to fill them in and send them by mail to the Chubu Clinical Oncology Group data center (Nagoya, Japan), which is independent from any of the hospitals. The clinical research coordinator at the data center checked patient eligibility with the registration form sent from the surgeon in charge of the patient and communicated with the patient thereafter regarding retrieval of the additional questionnaires at $1,3,6$, and 12 months following surgery. The surgeon reported on the surgical data including operating time, blood loss, lymph node retrieval, and morbidity, and pathological findings as soon as all the data were available, and the personnel at the data center decided whether to send further questionnaires based on the exclusion criteria.

The following patients were excluded from the study: those who were intraoperatively converted to open surgery because of unexpected findings of advanced gastric cancer, those who were converted to total gastrectomy for oncological reasons that were not predictable preoperatively (such as multiple cancer undetected preoperatively and positive surgical margin from unexpected cancerous infiltration). Those who were found at pathological examination of the resected specimens to have disease $\geq$ stage II were also excluded because the influence of adjuvant chemotherapy or disease recurrence was likely to interfere 
with detection of a difference in postoperative HRQOL. However, patients who were converted to total gastrectomy for technical reasons such as iatrogenic splenic injury were not excluded. In addition, those who were converted to open surgery because of any technical mishaps during surgery were to remain in the laparoscopic surgery group at analyses.

Pylorus-preserving gastrectomy (PPG) was performed when cancer occupied the middle third of the stomach and nodal metastasis seemed unlikely by preoperative imaging studies. D1+ dissection as defined in the Japanese Gastric Cancer Treatment Guidelines [12] was performed in clinically N0 cases and D2 dissection in clinically N1 cases. Cholecystectomy was indicated for those with cholelithiasis at the time of surgery. As a principle, the hepatic branch of the vagal nerve was preserved, but whether to preserve the celiac branch was left to the discretion of the surgeons. Billroth type I anastomosis was generally selected as a node of reconstruction, but Roux-en-Y anastomosis was conducted for patients with small gastric remnants.

The study was performed by ten participating hospitals affiliated to Nagoya University Graduate School of Medicine. Well-trained laparoscopic surgeons approved by the Japan Society for Endoscopic Surgery had been employed in four of the ten hospitals. In these hospitals, a majority of surgery for early-stage cancer is performed by the laparoscopic approach, and patients undergoing open approach were registered only when the patient wished to avoid laparoscopic surgery. In the other six hospitals, all surgery for early-stage cancer was performed by the open approach. Other affiliated hospitals that are in the process of introducing laparoscopic gastrectomy were discouraged from participating in the study because only lean patients with favorable characteristics are likely to undergo surgery by the laparoscopic approach. Surgeons are well accustomed to the technique needed to perform $\mathrm{D} 2$ dissection by open approach in all hospitals: the number of gastric cancer patients operated in the year 2009 were 199, 91, 79, and 66 in the four hospitals that performed laparoscopic surgery and 196, 90, 82, 81, 39, and 32 in the other six hospitals. The study was approved by the institutional review board in all hospitals.

\section{Statistical considerations}

The study was designed to have a power of 0.8 at a significance level of $5 \%$ to detect the difference in a physical functioning score of 5 points at 1 month postoperatively. Although the calculated sample size was 64 per group, accrual of 70 patients per group was planned to adjust for missing data.

Responses to the questionnaires were scored and missing data treated according to the EORTC scoring manual
[13]. Chi-squared analysis was used to compare categorical data, and Student's $t$ test was used for comparison of continuous variables. Linear mixed models were used for comparison of longitudinal scores for QLQ-C30 and QLQSTO22. For estimating the change in QOL scores from baseline, these models included surgical approach, baseline QOL score, time since the surgery, and the interaction term between surgical approach and time as covariates. A value of $p<0.05$ was considered to be statistically significant. Statistical analyses were performed using SAS version 9.2 (SAS Institute, Cary, NC, USA).

\section{Results}

Between September 2008 and January 2011, 159 patients were registered prospectively and preoperative score sheets were retrieved. Of these, 14 patients were deemed ineligible: 12 patients because of the final histopathological diagnosis of stage II disease, 1 patient because of subsequent total gastrectomy upon confirmation of cancer at the resection margin, and 1 patient because of body weight increase after registration that exceeded BMI of $28 \mathrm{~kg} / \mathrm{m}^{2}$. The remaining 145 patients underwent analyses; 72 underwent open surgery and 73 the laparoscopic approach. One patient who underwent laparoscopic distal gastrectomy was later treated by completion gastrectomy by open approach as a consequence of severe pancreatic fistula and related perforation of the remnant stomach but remained in the laparoscopic approach group for analysis. Six patients in the open surgery group and 10 patients in the laparoscopic surgery group underwent PPG. D2 lymph node dissection was performed in 12 patients among the open surgery group and 7 patients among the laparoscopic surgery group. Nine patients of the open group and 2 of the laparoscopic group underwent preservation of the celiac branch of the vagal nerve.

There were no significant differences between the groups regarding patient demographics and histopathological findings (Table 1). Reflecting the characteristics of gastric cancer patients in Japan, the mean age of the patients was relatively young (62.5 years for the laparoscopic group and 62.8 years for the open surgery group), with favorable BMI $\left(22.3 \pm 3.1 \mathrm{~kg} / \mathrm{m}^{2}\right.$ for the laparoscopic group and $22.4 \pm 2.7 \mathrm{~kg} / \mathrm{m}^{2}$ for the open surgery group). The laparoscopic approach required longer operating time $(230 \pm 52 \mathrm{~min}$ vs. $214 \pm 54 \mathrm{~min}, p=0.07)$ but the amount of blood loss was significantly less $(98.8 \pm 154.1 \mathrm{~g} \quad$ vs. $\quad 222.8 \pm 145.9 \mathrm{~g}, \quad p<0.0001)$ (Table 2). The number of lymph nodes retrieved was similar for both groups (33.5 for laparoscopic surgery and 31.0 for open surgery). Postoperative complications were observed in 7 patients treated by the laparoscopic approach 
( 2 cases of pancreatic fistula, 1 case each of anastomotic leakage, cholangitis, anastomotic stenosis, splenic infarction, and voiding disturbance) and 8 patients treated by open surgery ( 1 case of delayed gastric emptying and deep

Table 1 Comparison of patient characteristics between open gastrectomy (OG) group and laparoscopic gastrectomy (LAG) group

\begin{tabular}{|c|c|c|c|}
\hline & $\begin{array}{l}\text { OG group } \\
(n=72)\end{array}$ & $\begin{array}{l}\text { LAG group } \\
(n=73)\end{array}$ & $p$ value \\
\hline Gender (M/F) & $47 / 25$ & $51 / 22$ & 0.56 \\
\hline Mean age (range) (years) & $62.8(36-81)$ & $62.5(36-77)$ & 0.70 \\
\hline $\begin{array}{l}\text { Mean body mass index } \pm \text { SD } \\
\left(\mathrm{kg} / \mathrm{m}^{2}\right)\end{array}$ & $22.4 \pm 2.7$ & $22.3 \pm 3.1$ & 0.83 \\
\hline \multicolumn{4}{|l|}{ Macroscopic type } \\
\hline Type 0 & 71 & 72 & 0.99 \\
\hline Type 2 & 1 & 1 & \\
\hline \multicolumn{4}{|l|}{ Depth of invasion } \\
\hline $\mathrm{T} 1$ & 70 & 72 & 0.62 \\
\hline $\mathrm{T} 2$ & 2 & 1 & \\
\hline \multicolumn{4}{|l|}{ Lymph node metastasis } \\
\hline NO & 67 & 68 & 0.29 \\
\hline N1 & 3 & 5 & \\
\hline N2 & 2 & 0 & \\
\hline \multicolumn{4}{|l|}{ Pathological stage } \\
\hline I A & 65 & 67 & 0.78 \\
\hline I B & 7 & 6 & \\
\hline \multicolumn{4}{|l|}{ Pathological type } \\
\hline Differentiated & 32 & 41 & 0.34 \\
\hline Undifferentiated & 38 & 31 & \\
\hline Others & 2 & 1 & \\
\hline
\end{tabular}

vein thrombosis and 1 case each of intraabdominal abscess, anastomotic stenosis, anastomotic bleeding, wound infection, pseudomembranous colitis, delayed gastric emptying, and paralytic ileus), with no in-hospital mortality in either group.

No patient received postoperative adjuvant chemotherapy. One case in the open surgery group had metachronous cancer in the gastric remnant 17 months after surgery but it was removed by endoscopic submucosal dissection. Another case treated by open surgery committed suicide 19 months after surgery, and 1 case treated by laparoscopic surgery died of lung cancer 15 months after surgery. These patients were not excluded from the study.

The questionnaires were retrieved from $97.9 \%, 97.9 \%$, $97.9 \%, 93.8 \%$, and $95.9 \%$ of the patients at baseline, 1 month postoperatively, 3 months postoperatively, 6 months postoperatively, and 12 months postoperatively, respectively. The worst scores in most components of the questionnaire were observed at 1 month postoperatively and improved thereafter. Scores in the functioning scales recovered or surpassed the preoperative value by 12 months postoperatively, but several symptom scores remained inferior to the preoperative value (Fig. 1).

Analyses using the linear mixed model were conducted to detect differences between the two approaches throughout the 12 months of postoperative follow-up. No statistically significant differences were observed regarding physical functioning $(p=0.17)$, the primary endpoint, and global health status/QOL $(p=0.09)$. On the other hand, statistically significant differences in favor of the
Table 2 Comparison of surgical procedures and short-term outcomes between open gastrectomy (OG) group and laparoscopic gastrectomy (LAG) group
${ }^{\mathrm{a}} \mathrm{D} 1+$ is $\mathrm{D} 1$ with additional resection of some second-tier lymph nodes (nodes around the left gastric artery, common hepatic artery, and celiac axis)

\begin{tabular}{|c|c|c|c|}
\hline & OG group $(n=72)$ & LAG group $(n=73)$ & $p$ value \\
\hline \multicolumn{4}{|l|}{ Procedure for gastrectomy } \\
\hline Distal gastrectomy (DG) & 66 & 63 & \multirow[t]{2}{*}{0.42} \\
\hline Pylorus-preserving gastrectomy (PPG) & 6 & 10 & \\
\hline \multicolumn{4}{|l|}{ Lymph node dissection } \\
\hline $\mathrm{D} 1$ and $\mathrm{D} 1+^{\mathrm{a}}$ & 60 & 66 & \multirow[t]{2}{*}{0.31} \\
\hline D2 & 12 & 7 & \\
\hline \multicolumn{4}{|l|}{ Combined resection } \\
\hline Gallbladder & 6 & 3 & 0.33 \\
\hline \multicolumn{4}{|l|}{ Vagal nerve preservation } \\
\hline Abdominal branch & 9 & 2 & 0.031 \\
\hline \multicolumn{4}{|l|}{ Reconstruction procedure } \\
\hline B-I & 61 & 54 & \multirow[t]{4}{*}{0.31} \\
\hline B-II & 1 & 0 & \\
\hline Roux-en-Y & 5 & 9 & \\
\hline Gastro-gastro for PPG & 6 & 10 & \\
\hline No. of retrieval lymph nodes & $31.0 \pm 15.6$ & $33.5 \pm 13.5$ & 0.32 \\
\hline Operating time (min) & $213.8 \pm 53.8$ & $229.5 \pm 51.6$ & 0.07 \\
\hline Bleeding (g) & $222.8 \pm 145.9$ & $98.8 \pm 154.1$ & $<0.0001$ \\
\hline Operative morbidity & $8(11.1 \%)$ & $7(9.6 \%)$ & 0.79 \\
\hline
\end{tabular}



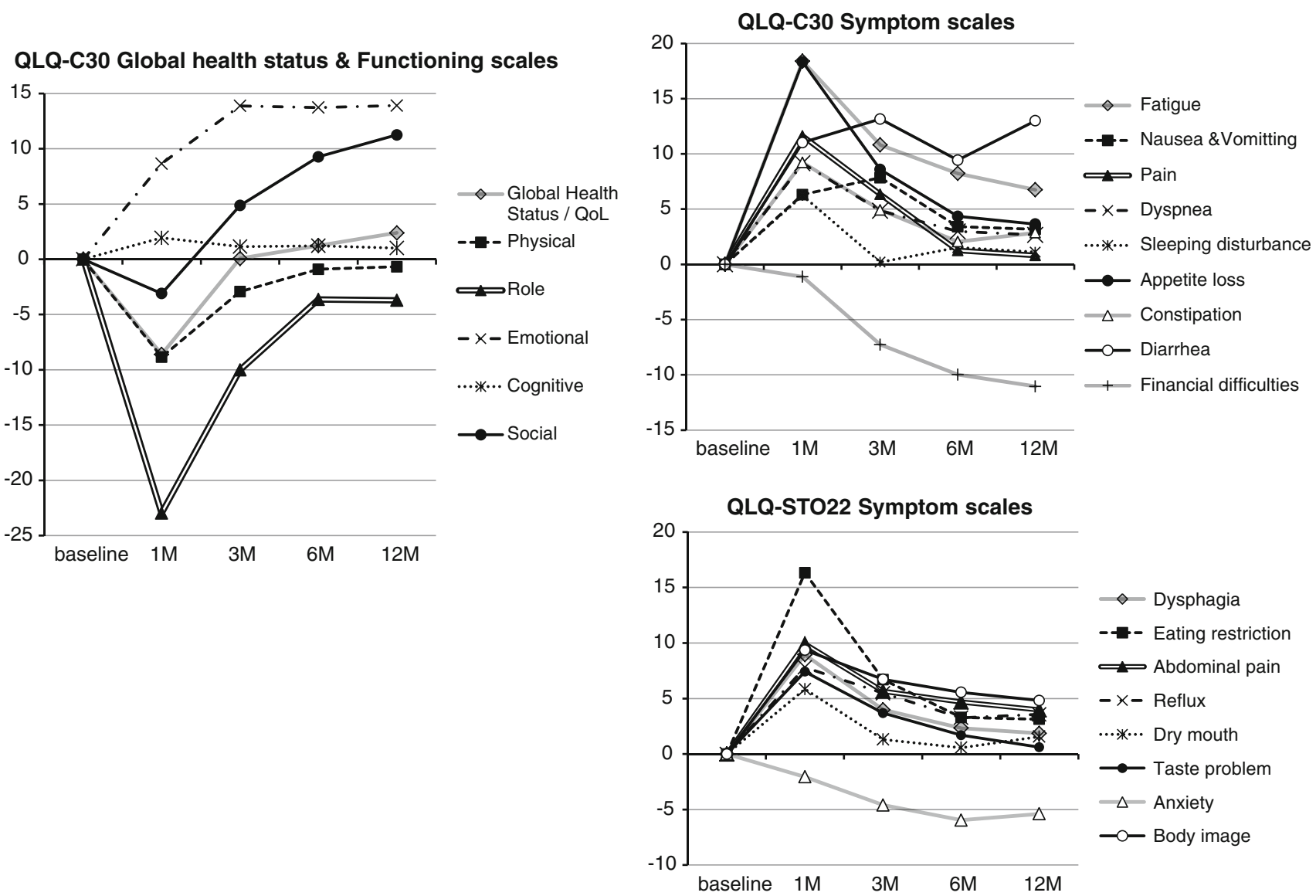

Fig. 1 Longitudinal changes in the mean scores of various components of the questionnaire among all patients during the first postoperative year. Scores for most components of the questionnaire showed the worst value at 1 month postoperatively and improved

laparoscopic approach were observed for role functioning ( $p=0.019)$, emotional functioning $(p=0.006)$, cognitive functioning $(p=0.033)$ and social functioning $(p=0.035)$ (Fig. 2). Of the three symptom scales and six single items in the QLQ-C30 reflecting postoperative symptoms, statistically significant differences in favor of the laparoscopic approach were observed in pain $(p=0.023)$ and fatigue $(p=0.036)$, although no difference was observed in the other seven components. Regarding the QLQ-STO22, statistically significant differences in favor of the laparoscopic approach were observed in eating restriction $(p=0.046)$, taste problems $(p=0.002)$, and anxiety $(p=0.036)$ (Fig. 3).

For the subscales in which significant differences were observed by analysis using the linear mixed model, comparisons were made between the two approaches at each time point after surgery. Scores for role functioning, emotional functioning, cognitive functioning, and social functioning showed a tendency to be superior for the laparoscopic approach at 6 and 12 months postoperatively. In contrast, all five symptom scales with significant

thereafter. A higher score represents superior outcome in the global health status and functioning scales. A lower score represents superior outcome in the symptom scales

differences in the previous analyses (two from the QLQ$\mathrm{C} 30$ and three from the QLQ-STO22) were shown to be in favor of the laparoscopic approach at 1,3, and 6 months postoperatively, whereas no difference was observed at 12 months postoperatively (Table 3 ).

\section{Discussion}

Safety and oncological outcomes such as survival time have been the traditional endpoints commonly used to evaluate treatments for cancer. More recently, patientoriented outcomes such as health-related quality of life (HRQOL) began to be recognized as additional endpoints in some types of clinical trials. Questionnaires that are to be used for the evaluation need to be well-established instruments in terms of reliability, reproducibility, feasibility, and clinical validity, rather than physician-based, non-validated questionnaires. The core questionnaire EORTC QLQ-C30 [8] and its supplementary organ-specific module QLQ-STO22 [9] were employed in the current 

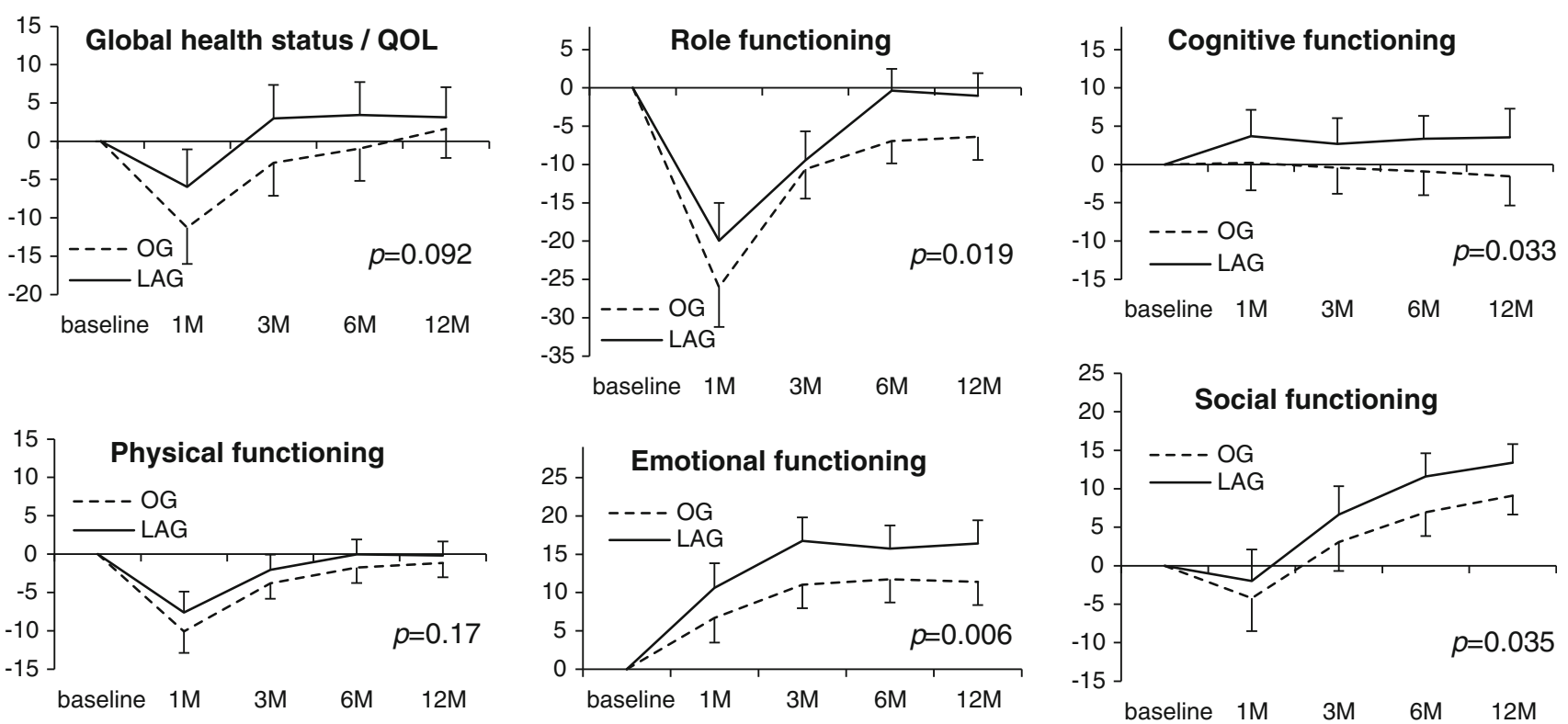

Fig. 2 Mean score changes from the baseline of the global health status and functioning scales of the open surgery group $(O G)$ and the laparoscopic surgery group $(L A G)$ at each time point. $Q O L$, quality of life; $M$, months. Error bars represent $95 \%$ confidence interval. Zero line represents the baseline score. Higher values denote superior outcome. $p$ values were calculated by the analyses using the linear mixed model to detect differences between the two approaches throughout the postoperative 12-month period
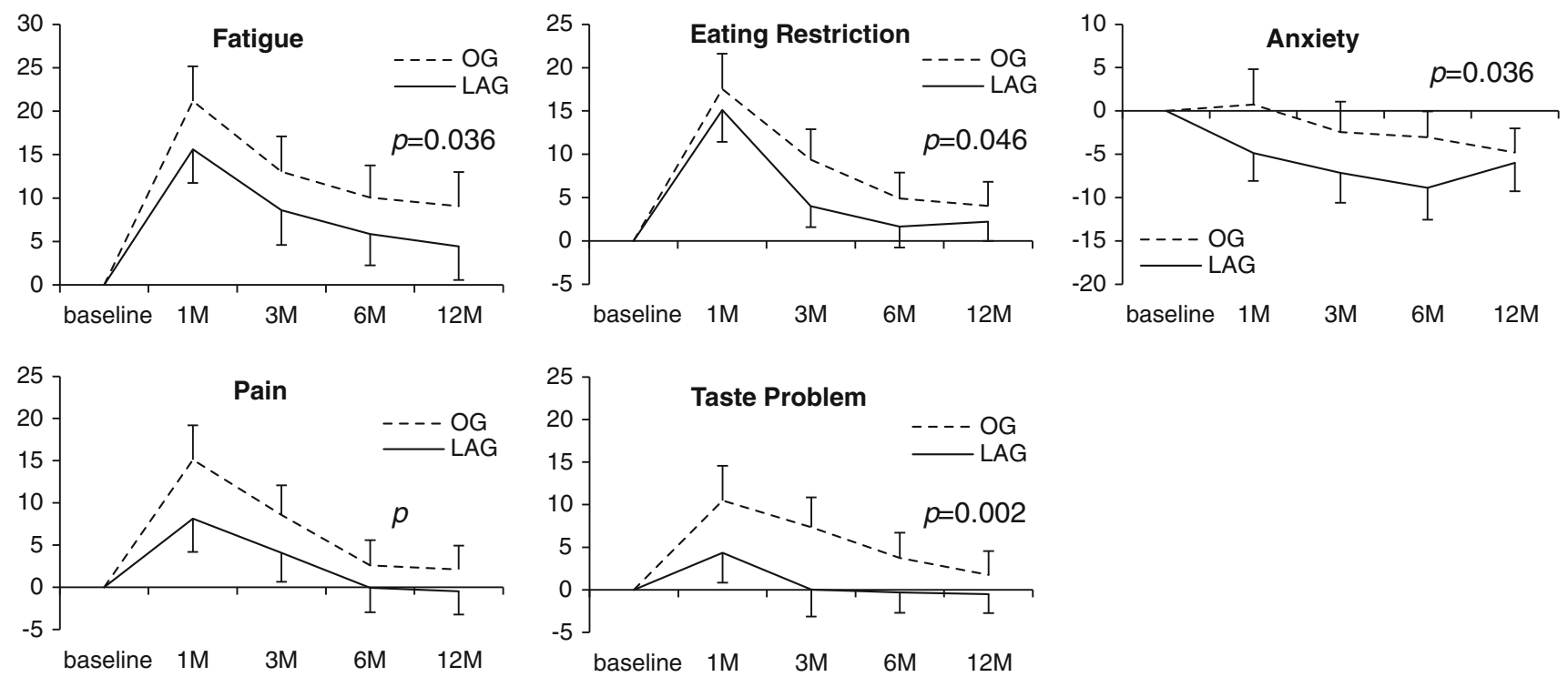

Fig. 3 Mean score changes from the baseline of five symptom scales in which significant differences between the two approaches were observed. Error bars represent $95 \%$ confidence interval. Zero line represents the baseline score. Lower values denote superior outcome.

study because these, along with FACT-G [14], have been translated into several languages and have undergone validation studies in the corresponding countries $[10,11$, $15]$.

In a previous study [16], the authors used the Japanese language version of the EORTC QLQ-C30 and STO22 to compare the postoperative HRQOL of patients who $p$ values were calculated by the analyses using the linear mixed model to detect differences between the two approaches throughout the postoperative 12-month period

underwent open total gastrectomy, open distal gastrectomy, and laparoscopic distal gastrectomy. A trend toward more favorable scores was observed among the patients treated by laparoscopic distal gastrectomy. However, because the laparoscopic approach was used more frequently to treat early-stage cancer, there were inevitable differences in background in that patients who were treated with open 
Table 3 Differences in mean scores between the two approaches and the corresponding $p$ values at each time point

\begin{tabular}{|c|c|c|c|c|c|c|c|c|}
\hline & \multicolumn{2}{|l|}{$1 \mathrm{M}$} & \multicolumn{2}{|l|}{$3 \mathrm{M}$} & \multicolumn{2}{|l|}{$6 \mathrm{M}$} & \multicolumn{2}{|l|}{$12 \mathrm{M}$} \\
\hline & Difference & $p$ value & Difference & $p$ value & Difference & $p$ value & Difference & $p$ value \\
\hline Role & -6.1 & $0.099 *$ & -1.2 & 0.67 & -6.5 & $0.002 * *$ & -5.4 & $0.014 * *$ \\
\hline Emotional & -3.9 & 0.10 & -5.7 & $0.012 * *$ & -4.0 & $0.074 *$ & -5.0 & $0.023 * *$ \\
\hline Cognitive & -3.5 & 0.18 & -3.1 & 0.21 & -4.3 & $0.057 *$ & -5.1 & $0.066^{*}$ \\
\hline Social & -2.3 & 0.46 & -2.6 & 0.19 & -4.7 & $0.036 * *$ & -4.3 & $0.016 * *$ \\
\hline Fatigue & 5.6 & $0.052 *$ & 4.4 & 0.13 & 4.2 & 0.12 & 4.6 & 0.10 \\
\hline Pain & 7.0 & $0.017 * *$ & 4.5 & $0.075^{*}$ & 2.7 & 0.21 & 2.6 & 0.20 \\
\hline Eating restriction & 2.4 & 0.37 & 5.4 & $0.003 * *$ & 3.2 & $0.070 *$ & 1.8 & 0.26 \\
\hline Taste problem & 6.2 & $0.018 * *$ & 7.3 & $0.002 * *$ & 4.1 & $0.023 * *$ & 2.3 & 0.16 \\
\hline Anxiety & 5.6 & $0.020 * *$ & 4.7 & $0.063^{*}$ & 5.8 & $\mathbf{0 . 0 3 3} * *$ & 1.2 & 0.63 \\
\hline
\end{tabular}

The difference was calculated as follows: (the mean score at the particular time point for OG - the base line score for OG) - (the mean score at the particular time point for LAG - the baseline score for LAG). Because high scores denote superior outcome for the functioning scales and inferior outcome for the symptom scales, negative values in the differences in functioning scales and positive values in the differences in symptom scales denote superiority of the laparoscopic approach. $M$ months

$*$ and italics mean $<0.1$

$* *$ and bold type mean $<0.05$

surgery had more advanced cancer and were liable to receive more extended lymph node dissection followed by postoperative adjuvant chemotherapy. To prove robustly that the laparoscopic approach results in improved HRQOL, the authors went on to perform the current prospective multi-institutional comparison between the two approaches only among patients who were diagnosed to have early ( $\mathrm{T} 1$ stage) gastric cancer.

The trend of longitudinal scores of all 145 patients revealed worst scores at 1 month after surgery in most of the subscales. The scores gradually improved and returned to the preoperative levels by 6 to 12 months postoperatively. However, some subscales did not assume this pattern. Subscales related to the mental status such as emotional functioning and anxiety had the worst value preoperatively. It is easy to imagine that even patients with early gastric cancer would feel anxious preoperatively, at the time when they had been informed of having a cancer and were awaiting surgery. These patients likely felt more relieved 1 month after surgery when they were informed that the early clinical stage was confirmed by pathological examination of the resected specimen after an uneventful postoperative course. However, the scores for fatigue and diarrhea among the symptom scales did not recover to the preoperative levels even after 12 months, as was found in the previous studies $[16,17]$. The fatigue score did improve after the worst score at the first postoperative month, but a period of 12 months was apparently not sufficient for full recovery. As for the diarrhea score, no improvement was observed throughout the first year. Diarrhea was presumably a result of early entry of food into the intestines, which induces hyperperistalsis, a symptom caused by the lack of a functional pyloric ring, and, again, is unlikely to improve in 12 months.

In the comparisons using the linear mixed model, no significant difference between the approaches was observed in physical functioning, the primary endpoint, where substantial difference had been detected in the previous study [16]. The smaller wound in the laparoscopic approach was expected to decrease stress caused by wound pain, and superiorities in symptoms scores such as pain and fatigue had actually been shown in the current study during the first 6 months. Our assumption was that these differences in symptoms would be translated into differences also in the physical functioning subscale, but this was not what we found. However, benefits of the less-invasive approach were actually shown in components such as role functioning and social functioning subscales. These results are encouraging signs for laparoscopic surgeons because these subscales are considered to more comprehensively reflect the patients' living status. On the other hand, no differences between the two approaches were observed in symptom scales related to the functional aspect of the digestive tract such as reflux, diarrhea, abdominal pain, and dysphagia. These results had been anticipated because, theoretically, no difference regarding symptoms caused by the loss of the distal stomach should exist between the two approaches.

The strength of this trial is in the comparison of the HRQOL measured longitudinally at 1, 3, 6, and 12 months after surgery, which provides information regarding how long the seemingly beneficial effect of minimally invasive surgery actually persists. In most of the previous studies, comparisons between the approaches had been made only 
at one time point and at varying time intervals after surgery. Yasuda et al. [18] used their own questionnaires to compare HRQOL, which was measured at a single time point (mean time interval from surgery, 98 months) and found no statistical difference between the approaches. Ikenaga et al. [19] also used their original questionnaire and compared the data measured at a single time point (mean time interval from surgery, 35 months). The patients who underwent LAG tended to be more satisfied, but benefit after a long-term follow-up was not clarified. Liu et al. [20] used EORTC QLQ-C30 and STO22 and reported superior HRQOL of the LAG at a mean follow-up time of 21 months. Lee et al. [21] also used EORTC QLQ-C30 and STO22 and reported inferior HRQOL of the LAG at a mean follow-up time of 22 months. In all these studies, the time point for evaluation of the HRQOL had not been prespecified, and moreover the data at baseline (before surgery) were unavailable.

Kim et al. [22] performed a prospective randomized study to explore the same issue using the same instruments and found no significant difference in most subscales from 6 months to 5 years after surgery, despite the significant advantage in favor of LAG up to 3 months postoperatively [23]. This study has shown quite convincingly that the laparoscopic approach improves QOL only during the early postoperative recovery when patients are estimated to benefit most from the smaller wound and less pain characteristic of the minimally invasive approach. However, there are weaknesses in this study in addition to being a single-institution study in which all surgery was conducted by a single surgeon. The linear mixed model comprehensively compares the health-related outcome scores between the two approaches during the period from 6 months to 5 years postoperatively. This comparison could overlook or underestimate any possible difference that may be found relatively early at 6 to 12 months after surgery. The aim of our study was to explore whether the well-documented benefits of LAG could persist beyond the first 6 months. We have considered the differences between the two approaches for up to 12 months postoperatively and identified subscales in which significant differences were detected by the linear mixed model. For these subscales, comparisons were also conducted at each time point by Student's $t$ test, and significant differences in favor of the LAG was observed for role, social, and emotional functioning scores even at 12 months after surgery. Thus, our results point to the encouraging fact that the benefit of the minimally invasive approach is not necessarily transient.

A weakness inherent to this study is that it is not a randomized trial. Although LAG for early-stage cancer has been covered by insurance in Japan and is often selected in general clinical practice to treat early-stage cancer, even in community hospitals, it has not been listed as a standard treatment in the Japanese guidelines for the treatment of gastric cancer [12]. Under the circumstances in which the newer treatment option is no longer perceived by the public as purely investigational, it was not practical for a study group consisting mainly of community hospitals to conduct a randomized trial. To minimize biases, institutions where surgeons were apparently in the learning-curve phase for LAG were not invited to participate, whereas all participating institutions performed a sufficient number of gastrectomies per year to be considered as high volume by the Western standard [24, 25]. Another weakness is the lack of data beyond 12 months. However, Kim et al. [22] suggested in a randomized trial that analysis of data beyond that time point could be futile. A pivotal randomized trial, JCOG0912, comparing the two approaches for early-stage gastric cancer with overall survival as a primary endpoint has recently completed patient accrual [26]. In this study, assessment of HRQOL, one of the secondary endpoints, has been conducted at the baseline and at 1, 3, and 12 months and 3 years after surgery, and will eventually provide convincing data regarding the difference in HRQOL in the long term. From what we have learned from the current study, the future trial to explore benefits of the minimally invasive approach in terms of QOL should conduct assessment for at least 12 months and select an endpoint that more comprehensively observes patient living status such as role functioning and social functioning subscales. In addition, the use of novel questionnaires to evaluate postgastrectomy symptoms in greater detail [27] needs to be seriously considered. However, final results and interpretations of the JCOG0912 might rule out the possibility of conducting further open versus laparoscopic randomized trials in early-stage cancer. If such is the case, future trials will need to select more advanced cancer as a target.

To conclude, laparoscopic distal gastrectomy for earlystage cancer can be conducted safely by surgeons with sufficient expertise. The study was considered negative because no benefit of laparoscopic approach was observed in terms of physical functioning, the primary endpoint, throughout the 12 months postoperatively. However, the minimally invasive approach resulted in more favorable scores for some of the symptom scales during the first 6 months, and benefits in terms of several functioning scales were detectable even at 12 months after surgery.

\section{References}

1. Fleshman J, Sargent DJ, Green E, Anvari M, Stryker SJ, Beart RW Jr, Hellinger M, Flanagan R Jr, Peters W, Nelson H. Laparoscopic colectomy for cancer is not inferior to open surgery based on 5-year data from the COST study group trial. Ann Surg. 2007;246(4):655-62. 
2. Buunen M, Veldkamp R, Hop WC, Kuhry E, Jeekel J, Haglind E, Pahlman L, Cuesta MA, Msika S, Morino M, et al. Survival after laparoscopic surgery versus open surgery for colon cancer: longterm outcome of a randomised clinical trial. Lancet Oncol. 2009;10(1):44-52.

3. Etoh T, Inomata M, Shiraishi N, Kitano S. Minimally invasive approaches for gastric cancer-Japanese experiences. J Surg Oncol. 2013;107(3):282-8.

4. Hosono S, Arimoto Y, Ohtani H, Kanamiya Y. Meta-analysis of short-term outcomes after laparoscopy-assisted distal gastrectomy. World J Gastroenterol. 2006;12(47):7676-83.

5. Kitano S, Shiraishi N, Uyama I, Sugihara K, Tanigawa N. A multicenter study on oncologic outcome of laparoscopic gastrectomy for early cancer in Japan. Ann Surg. 2007;245(1):68-72.

6. Kim HH, Hyung WJ, Cho GS, Kim MC, Han SU, Kim W, Ryu SW, Lee HJ, Song KY. Morbidity and mortality of laparoscopic gastrectomy versus open gastrectomy for gastric cancer: an interim report-a phase III multicenter, prospective, randomized trial (KLASS trial). Ann Surg. 2010;251(3):417-20.

7. Katai H, Sasako M, Fukuda H, Nakamura K, Hiki N, Saka M, Yamaue H, Yoshikawa T, Kojima K. Safety and feasibility of laparoscopy-assisted distal gastrectomy with suprapancreatic nodal dissection for clinical stage I gastric cancer: a multicenter phase II trial (JCOG 0703). Gastric Cancer. 2010;13(4):238-44.

8. Aaronson NK, Ahmedzai S, Bergman B, Bullinger M, Cull A, Duez NJ, Filiberti A, Flechtner H, Fleishman SB, de Haes JC, et al. The European Organization for Research and Treatment of Cancer QLQ-C30: a quality-of-life instrument for use in international clinical trials in oncology. $J$ Natl Cancer Inst. 1993;85(5):365-76.

9. Blazeby JM, Conroy T, Bottomley A, Vickery C, Arraras J, Sezer O, Moore J, Koller M, Turhal NS, Stuart R, et al. Clinical and psychometric validation of a questionnaire module, the EORTC QLQ-STO 22, to assess quality of life in patients with gastric cancer. Eur J Cancer. 2004;40(15):2260-8.

10. Kobayashi K, Takeda F, Teramukai S, Gotoh I, Sakai H, Yoneda S, Noguchi Y, Ogasawara H, Yoshida K. A cross-validation of the European Organization for Research and Treatment of Cancer QLQ-C30 (EORTC QLQ-C30) for Japanese with lung cancer. Eur J Cancer. 1998;34(6):810-5.

11. Morita S, Kaptein AA, Oba K, Sakamoto J. The domain structure of the EORTC QLQ-STO22 supported by Japanese validation data. Psychooncology. 2008;17(5):474-9.

12. Japanese Gastric Cancer Association. Japanese gastric cancer treatment guidelines 2010 (ver. 3). Gastric Cancer. 2011;14(2): 113-23.

13. Fayers P, Aaronson N, Bjordal K, Groenvold M, Curran D, Bottomley A. EORTC QLQ-C30 scoring manual. 3rd ed. Brussels: European Organization for Research and Treatment of Cancer; 2001.

14. Cella DF, Tulsky DS, Gray G, Sarafian B, Linn E, Bonomi A, Silberman M, Yellen SB, Winicour P, Brannon J, et al. The Functional Assessment of Cancer Therapy scale: development and validation of the general measure. $J$ Clin Oncol. 1993;11(3):570-9.

15. Fumimoto H, Kobayashi K, Chang CH, Eremenco S, Fujiki Y, Uemura S, Ohashi Y, Kudoh S. Cross-cultural validation of an international questionnaire, the General Measure of the
Functional Assessment of Cancer Therapy scale (FACT-G), for Japanese. Qual Life Res. 2001;10(8):701-9.

16. Kobayashi D, Kodera Y, Fujiwara M, Koike M, Nakayama G, Nakao A. Assessment of quality of life after gastrectomy using EORTC QLQ-C30 and STO22. World J Surg. 2011;35(2): 357-64.

17. Kong $\mathrm{H}$, Kwon $\mathrm{OK}, \mathrm{Yu}$ W. Changes of quality of life after gastric cancer surgery. J Gastric Cancer. 2012;12(3):194-200.

18. Yasuda K, Shiraishi N, Etoh T, Shiromizu A, Inomata M, Kitano S. Long-term quality of life after laparoscopy-assisted distal gastrectomy for gastric cancer. Surg Endosc. 2007;21(12): 2150-3.

19. Ikenaga N, Nishihara K, Iwashita T, Suehara N, Mitsuyama S. Long-term quality of life after laparoscopically assisted distal gastrectomy for gastric cancer. J Laparoendosc Adv Surg Tech A. 2006;16(2):119-23.

20. Liu J, Yang K, Chen XZ, Dai B, Zhang B, Chen ZX, Chen JP, Zhou ZG, Hu JK. Quality of life following laparoscopic-assisted distal gastrectomy for gastric cancer. Hepatogastroenterology. 2012;59(119):2207-12.

21. Lee SS, Ryu SW, Kim IH, Sohn SS. Quality of life beyond the early postoperative period after laparoscopy-assisted distal gastrectomy: the level of patient expectation as the essence of quality of life. Gastric Cancer. 2012;15(3):299-304.

22. Kim YW, Yoon HM, Yun YH, Nam BH, Eom BW, Baik YH, Lee SE, Lee Y, Kim YA, Park JY, et al. Long-term outcomes of laparoscopy-assisted distal gastrectomy for early gastric cancer: result of a randomized controlled trial (COACT 0301). Surg Endosc. 2013;27(11):4267-76.

23. Kim YW, Baik YH, Yun YH, Nam BH, Kim DH, Choi IJ, Bae JM. Improved quality of life outcomes after laparoscopy-assisted distal gastrectomy for early gastric cancer: results of a prospective randomized clinical trial. Ann Surg. 2008;248(5):721-7.

24. Dikken JL, Dassen AE, Lemmens VE, Putter H, Krijnen P, van der Geest L, Bosscha K, Verheij M, van de Velde CJ, Wouters MW. Effect of hospital volume on postoperative mortality and survival after oesophageal and gastric cancer surgery in the Netherlands between 1989 and 2009. Eur J Cancer. 2012;48(7):1004-13.

25. Anderson O, Ni Z, Moller H, Coupland VH, Davies EA, Allum WH, Hanna GB. Hospital volume and survival in oesophagectomy and gastrectomy for cancer. Eur J Cancer. 2011;47(16): $2408-14$

26. Nakamura K, Katai H, Mizusawa J, Yoshikawa T, Ando M, Terashima M, Ito S, Takagi M, Takagane A, Ninomiya M, et al. A phase III study of laparoscopy-assisted versus open distal gastrectomy with nodal dissection for clinical stage IA/IB gastric cancer (JCOG0912). Jpn J Clin Oncol. 2013;43(3):324-7.

27. Nakada K, Ikeda M, Takahashi M, Kinami S, Yoshida M, Uenosono Y, Kawashima Y, Oshio A, Suzukamo Y, Terashima M, Kodera Y. Characteristics and clinical relevance of postgastrectomy syndrome assessment scale (PGSAS)-45: newly developed integrated questionnaires for assessment of living status and quality of life in postgastrectomy patients. Gastric Cancer 2014. doi:10.1007/s10120-014-0344-4 